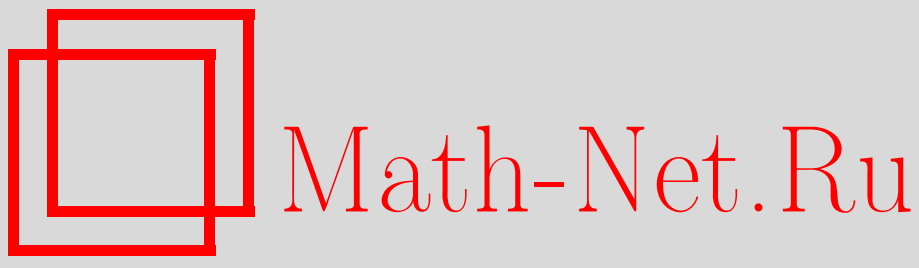

А. С. Ерёмин, Композиция смешанного дробного интеграла и смешанной дробной производной Римана-Лиувилля одного порядка, Вестн. Сам. гос. техн. ун-та. Сер. Физ.-мат. науки, 2005, выпуск 34, 16-24

DOI: https://doi.org/10.14498/vsgtu333

Использование Общероссийского математического портала Math-Net.Ru подразумевает, что вы прочитали и согласны с пользовательским соглашением

http://www . mathnet.ru/rus/agreement

Параметры загрузки:

IP: 54.237 .206 .68

26 апреля 2023 г., 17:41:57

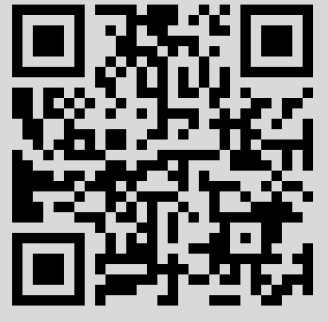


дробной производной по времени // Мат. моделирование и краевые задачи. Тр. тринадцатой межвуз. конференции. Часть 3. Самара: СамГТУ, 2003. С. 185-186

17. Азовский В.В., Носов В.А. Решение обобщенной задачи Трикоми для одного уравнения смешанного типа в бесконечной области // Волжский математический сборник. Куйбышев: Куйб. пед. ин-т. Вып. 15, 1973. С. 3-9.

18. Свешников А.Г., Тихонов А.Н. Теория функций комплексной переменной. М.: Наука, 1979. 320 с.

19. Бицадзе A.B. К проблеме уравнений смешанного типа // Тр. мат. ин-та им. Стеклова. М.: Наука, 41. 1953. 62 с.

Поступила 24.01.2005 2.

УДК 517.95

А.С. Еремин

\section{КОМПОЗИЦИЯ СМЕШАННОГО ДРОБНОГО ИНТЕГРАЛА И СМЕШАННОЙ ДРОБНОЙ ПРОИЗВОДНОЙ РИМАНА-ЛИУВИЛЛЯ ОДНОГО ПОРЯДКА}

Исследуется вопрос композици смешанного дробного интеграла и смешанной дробной производной в достаточно широком классе функиий. Получена формула обращения смешанной дробной производной.

Введение Теория дробного исчисления посвящена исследованию и применению интегралов и производных произвольного порядка. Историю дробного исчисления следует вести еще с работ Н. Абеля и Ж. Лиувилля [1]. В последнее время интерес к дробному исчислению значительно усилился, что вызвано многочисленными приложениями в различных областях науки. В этой связи можно упомянуть монографии [2-6], работы [7-9].

Известны различные формы дробных интегралов и производных. Наиболее часто в научной литературе встречаются дробные интегралы и производные Римана-Лиувилля [1]. Операторы обобщенного дробного интегро-дифференцирования с гипергеометрической функцией Гаусса $F(a ; b ; c ; z)$ рассматриваются, например, в работах [10-13]. В работах [14-16] операторы дробного интегро-дифференцирования обобщаются на случай матричного интегродифференцирования.

Непосредственное распространение операций дробного интегро-дифференцирования Римана-Лиувилля на случай многих переменных, когда эти операторы применяются по каждой переменной или по некоторым из них, дает так называемые частные и смешанные дробные интегралы и производные. Они известны достаточно давно [1]. Так, в работе [17] при помощи двухмерного преобразования Лапласа получено решение двухмерного интегрального уравнения Абеля.

В настоящей работе исследуется вопрос композиции смешанного дробного интеграла и смешанной дробной производной в достаточно широком классе функций. Получена формула обращения смешанной дробной производной. Полученные результаты могут применяться в теории дифференциальных уравнений, содержащих смешанные дробные производные.

Абсолютно непрерывные функции. Важную роль в теории дробного интегродифференцирования играют абсолютно непрерывные функции.

Пусть $\Omega=\{(x, y): a<x<b, c<y<d\},-\infty \leq a<b \leq \infty,-\infty \leq c<d \leq \infty$.

Определение 1 [1, с. 21]. Функция $f(x)$ называется абсолютно непрерывной на отрезке $[a, b]$, если по любому $\varepsilon>0$ можно найти такое $\delta>0$, что для любой конечной системы попарно непересекающихся отрезков $\left[a_{k}, b_{k}\right] \in[a, b], k=\overline{1, m}$, такой, что $\sum_{k=1}^{m}\left(b_{k}-a_{k}\right)<\delta$, справедливо неравенство $\sum_{k=1}^{m}\left|f\left(b_{k}\right)-f\left(a_{k}\right)\right|<\varepsilon$. Класс всех таких функций обозначается $A C([a, b])$.

Определение 2 [1, с. 21]. Через $A C^{n}([a, b])$, где $n=1,2, \ldots$ обозначим класс функций $f(x)$, непрерывно дифференцируемых на $[a, b]$ до порядка $n-1$, причем $f^{(n-1)}(x) \in A C([a, b])$. Определение 3 [18, с. 237]. Функция $f(x, y)$ называется абсолютно непрерывной в $\Omega$, если по любому $\varepsilon>0$ можно найти такое $\delta>0$, что для любой конечной системы попарно непересекающихся промежутков $\Delta_{k}=\left\{(x, y): x_{1 k} \leq x \leq x_{2 k}, y_{1 k} \leq y \leq y_{2 k}\right\}$, сумма площадей которых 
меньше $\delta$, справедливо неравенство

$$
\sum_{k=1}^{n}\left|f\left(x_{2 k}, y_{2 k}\right)-f\left(x_{1 k}, y_{2 k}\right)-f\left(x_{2 k}, y_{1 k}\right)+f\left(x_{1 k}, y_{1 k}\right)\right|<\varepsilon,
$$

и если, кроме того, $f(a, y) \in A C([c, d])$ и $f(x, c) \in A C([a, b])$. Класс всех таких функций обозначается $A C(\bar{\Omega})$.

Определение 4. Через $A C^{n, m}(\bar{\Omega})$, где $n=1,2, \ldots$, обозначим класс функций, непрерывно дифференцируемых на $\bar{\Omega}$ до порядка $(n-1, m-1)$, причем ее смешанная частная производная $\partial^{n+m-2} f / \partial x^{n-1} \partial y^{m-1}$ абсолютно непрерывна в $\bar{\Omega}$.

Известно [19, с. 338], что классу $A C([a, b])$ принадлежат те и только те функции $f(x)$, которые представимы в виде первообразных от суммируемых по Лебегу функций:

$$
f(x)=\int_{a}^{x} \psi(x) d x+c, \quad \psi(x) \in L_{1}(a, b) .
$$

Лемма $1[1$, c. 46$]$. Классу $A C^{n}([a, b])$ принадлежат те и только те функции $f(x)$, которые представимы в виде

$$
f(x)=\frac{1}{(n-1) !} \int_{a}^{x}(x-t)^{n-1} \varphi(t) d t+\sum_{k=0}^{n-1} c_{k}(x-a)^{k},
$$

где $\varphi(x) \in L_{1}([a, b])$, а $c_{k}$ - произвольные постоянные.

В формуле (3)

$$
\varphi(t)=f^{(n)}(t), \quad c_{k}=f^{(k)}(a) / k ! .
$$

В последнем равенстве используется обозначение $f^{(n)}(x)=d^{n} f(x) / d x^{n}$.

Аналогичное свойство функций $f(x, y) \in A C(\bar{\Omega})$ выглядит следующим образом.

Лемма $2[18$, с. 238$]$. Классу $A C(\bar{\Omega})$ принадлежат те и только те функции $f(x, y)$, которые представимы в виде

$$
f(x, y)=\int_{a}^{x} \int_{c}^{y} \varphi(x) d x d y+\int_{a}^{x} \psi(x) d x+\int_{c}^{y} \xi(y) d y+d,
$$

где $\varphi(x, y) \in L_{1}(\Omega), \psi(x) \in L_{1}(a, b), \xi(y) \in L_{1}(c, d)$, a $d$ - произвольная постоянная.

Для того чтобы обобщить последнюю лемму на случай класса $A C^{n, m}(\bar{\Omega})$, нам понадобится следующая лемма.

Лемма 3. Пусть $f(x, y) \in A C^{n, m}(\bar{\Omega})$, тогда справедлива формула

$$
\begin{gathered}
f(x, y)=\frac{1}{(n-1) !(m-1) !} \int_{a}^{x} \int_{c}^{y}(x-t)^{n-1}(y-s)^{m-1} f^{(n, m)}(x, y) d x d y+ \\
+\sum_{i=0}^{n-1} \frac{f^{(i, 0)}(a, y)}{i !}(x-a)^{i}+\sum_{k=0}^{m-1} \frac{f^{(0, k)}(x, c)}{k !}(y-c)^{k}-\sum_{i=0}^{n-1} \sum_{k=0}^{m-1} \frac{f^{(i, k)}(a, c)}{i ! k !}(x-a)^{i}(y-c)^{k} .
\end{gathered}
$$

В формуле (6) использовано обозначение $f^{(i, k)}(x, y)=d^{i+k} f(x, y) / d x^{i} d y^{k}$.

Доказательство. Пусть $\frac{\partial^{n+m-2} f}{\partial x^{n-1} \partial y^{m-1}} \in A C(\bar{\Omega})$. В силу леммы 2 имеем

$$
\frac{\partial^{n+m-2} f}{\partial x^{n-1} \partial y^{m-1}}=\int_{a}^{x} \int_{c}^{y} \varphi(x) d x d y+\int_{a}^{x} \psi(x) d x+\int_{c}^{y} \xi(y) d y+d_{0} .
$$

Интегрируя последовательно (7) $n-1$ раз по $x$ и $m-1$ раз по $y$, получим

$$
\begin{aligned}
f(x, y)= & \frac{1}{(n-1) !(m-1) !} \int_{a}^{x} \int_{c}^{y}(x-t)^{n-1}(y-s)^{m-1} \varphi(x, y) d x d y+\frac{(y-c)^{m-1}}{(n-1) !(m-1) !} \int_{a}^{x}(x-t)^{n-1} \psi(x) d x+ \\
& +\frac{(x-a)^{n-1}}{(n-1) !(m-1) !} \int_{c}^{y}(y-c)^{m-1} \xi(y) d y+\sum_{i=0}^{n-1} \overline{\tau_{i}}(y)(x-a)^{i}+\sum_{k=0}^{m-1} \tilde{\tau}_{k}(x)(y-c)^{k},
\end{aligned}
$$

где $\overline{\tau_{i}}(y) \quad(i=\overline{0, n-1}), \tilde{\tau}_{k}(x)(k=\overline{0, m-1})-$ произвольные функции. При интегрировании использована широко известная для $n$-кратного интеграла формула [1] 


$$
\int_{a}^{x} d x \int_{a}^{x} d x \ldots \int_{a}^{x} F(x) d x=\frac{1}{(n-1) !} \int_{a}^{x}(x-t)^{n-1} F(t) d t
$$

доказательство которой легко осуществить методом математической индукции. Из доказательства станет ясно, что произвольная постоянная в формуле (7) связана с произвольными функциями формулы (8) соотношением

$$
(n-1) ! !^{-(m-1)}(c)+(m-1) ! \tilde{\tau}_{m-1}^{(n-1)}(a)=d_{0} .
$$

Поскольку $f(x, y) \in A C^{n, m}(\bar{\Omega})$, то производные $\frac{\partial^{i+k} f}{\partial x^{i} \partial y^{k}} \quad(0 \leq i<n, 0 \leq k<m)$ существуют и непрерывны в $\Omega$. Вычисляя производные по $x$ порядка $0, \ldots, n-1$ функции $f(x, y)$, задаваемой формулой (8), и полагая в них $x=a$, получим равенства

$$
\begin{gathered}
\frac{\partial^{i} f(a, y)}{\partial x^{i}}=i ! \bar{\tau}_{i}(y)+\sum_{k=0}^{m-1} \tilde{\tau}_{k}^{(i)}(a)(y-c)^{k}, \quad i=\overline{0, n-2}, \\
\frac{\partial^{n-1} f(a, y)}{\partial x^{n-1}}=\frac{1}{(m-1) !} \int_{c}^{y}(y-c)^{m-1} \xi(y) d y+(n-1) ! \bar{\tau}_{n-1}(y)+\sum_{k=0}^{m-1} \tilde{\tau}_{k}^{(n-1)}(a)(y-c)^{k} .
\end{gathered}
$$

Аналогичным образом, дифференцируя (8) по $y$ и полагая $y=c$, получим равенства

$$
\begin{gathered}
\frac{\partial^{k} f(x, c)}{\partial y^{k}}=\sum_{i=0}^{n-1} \bar{\tau}_{i}^{(k)}(c)(x-a)^{i}+k ! \tilde{\tau}_{k}(x), \quad k=\overline{0, m-2}, \\
\frac{\partial^{m-1} f(x, c)}{\partial y^{m-1}}=\frac{1}{(n-1)} \int_{a}^{x}(x-t)^{n-1} \psi(x) d x+\sum_{i=0}^{n-1} \bar{\tau}_{i}^{-(m-1)}(c)(x-a)^{i}+(m-1) \tilde{\tau}_{m-1}(x) .
\end{gathered}
$$

Выражая из формул $(10)-(13) \bar{c}_{i}(y)$ и $\tilde{c}_{i}(x)$ соответственно, получим

$$
\begin{aligned}
& \sum_{i=0}^{n-1} \bar{\tau}_{i}(y)(x-a)^{i}+\sum_{k=0}^{m-1} \tilde{\tau}_{k}(x)(y-c)^{k}= \\
& =\sum_{i=0}^{n-1} \frac{(x-a)^{i}}{i !}\left(\frac{\partial^{i} f(a, y)}{\partial x^{i}}-\sum_{k=0}^{m-1} \tilde{\tau}_{k}^{(i)}(a)(y-c)^{k}\right)+\sum_{k=0}^{m-1}(y-c)^{k}\left(\frac{\partial^{k} f(x, c)}{\partial y^{k}}-\sum_{i=0}^{n-1} \bar{\tau}_{i}^{-(k)}(c)(x-a)^{i}\right)- \\
& -\frac{(x-a)^{n-1}}{(n-1) !(m-1) !} \int_{c}^{y}(y-c)^{m-1} \xi(y) d y-\frac{(y-c)^{m-1}}{(n-1) !(m-1) !} \int_{a}^{x}(x-t)^{n-1} \psi(x) d x= \\
& =\sum_{i=0}^{n-1} \frac{(x-a)^{i}}{i !} \frac{\partial^{i} f(a, y)}{\partial x^{i}}+\sum_{k=0}^{m-1}(y-c)^{k} \frac{\partial^{k} f(x, c)}{\partial y^{k}}- \\
& -\frac{(x-a)^{n-1}}{(n-1) !(m-1) !} \int_{c}^{y}(y-c)^{m-1} \xi(y) d y-\frac{(y-c)^{m-1}}{(n-1) !(m-1) !} \int_{a}^{x}(x-t)^{n-1} \psi(x) d x- \\
& -\sum_{i=0}^{n-1} \sum_{k=0}^{m-1}(x-a)^{i}(y-c)^{k}\left(\frac{\tau_{i}^{(k)}(c)}{k !}+\frac{\tilde{\tau}_{k}^{(i)}(a)}{i !}\right) .
\end{aligned}
$$

Вычисляя смешанные производные $\frac{\partial^{i+k} f}{\partial x^{i} \partial y^{k}}$ функции (8) в точке $(a, c)$, получим

$$
\frac{1}{i ! k !} \frac{\partial^{i+k} f(a, c)}{\partial x^{i} \partial y^{k}}=\frac{\bar{\tau}_{i}^{(k)}(c)}{k !}+\frac{\tilde{\tau}_{k}^{(i)}(a)}{i !} .
$$

Подставляя (14), (15) в (8), получим

$$
\begin{gathered}
f(x, y)=\frac{1}{(n-1) !(m-1) !} \int_{a}^{x} \int_{c}^{y}(x-t)^{n-1}(y-s)^{m-1} \varphi(x, y) d x d y+ \\
+\sum_{i=0}^{n-1} \frac{1}{i !} \frac{\partial^{i} f(a, y)}{\partial x^{i}}(x-a)^{i}+\sum_{k=0}^{m-1} \frac{1}{k !} \frac{\partial^{k} f(x, c)}{\partial y^{k}}(y-c)^{k}-\sum_{i=0}^{n-1} \sum_{k=0}^{m-1} \frac{1}{i ! k !} \frac{\partial^{i+k} f(a, c)}{\partial x^{i} \partial y^{k}}(x-a)^{i}(y-c)^{k} .
\end{gathered}
$$

Равенство (6) следует из (16) и из того факта, что $\varphi(x, y)=\frac{\partial^{m+n} f(a, c)}{\partial x^{n} \partial y^{m}}$. Лемма доказана.

Следующая лемма дает описание класса $A C^{n, m}(\bar{\Omega})$. Она обобщает лемму 1 на случай двух 
переменных и лемму 2 на случай $n+m>2$.

Лемма 4. Классу $A C^{n, m}(\bar{\Omega})$ принадлежат те и только те функции $f(x, y)$, которые представимы в виде

$$
\begin{gathered}
f(x, y)=\frac{1}{(n-1) !(m-1) !} \int_{a}^{x} \int_{c}^{y}(x-t)^{n-1}(y-s)^{m-1} \varphi(x, y) d x d y+ \\
+\sum_{k=0}^{m-1} \frac{(y-c)^{k}}{k !(n-1) !} \int_{a}^{x}(x-t)^{n-1} \psi_{k}(x) d x+\sum_{i=0}^{n-1} \frac{(x-a)^{i}}{i !(m-1) !} \int_{c}^{y}(y-c)^{m-1} \xi_{i}(y) d y+\sum_{i=0}^{n-1} \sum_{k=0}^{m-1} d_{i k}(x-a)^{i}(y-c)^{k},
\end{gathered}
$$

где $\varphi(x, y) \in L_{1}(x, y), \quad \psi_{k}(x) \in L_{1}(a, b) \quad(k=\overline{0, m-1}), \quad \xi_{i}(y) \in L_{1}(c, d)(i=\overline{0, n-1})$, а $d_{i k}$ - произвольные постоянные.

Доказательство. Необходимость. Пусть $f(x, y) \in A C^{n, m}(\bar{\Omega})$. Согласно лемме 3

$$
\begin{gathered}
f(x, y)=\frac{1}{(n-1) !(m-1) !} \int_{a}^{x} \int_{c}^{y}(x-t)^{n-1}(y-s)^{m-1} f^{(n, m)}(x, y) d x d y+ \\
+\sum_{i=0}^{n-1} \frac{f^{(i, 0)}(a, y)}{i !}(x-a)^{i}+\sum_{k=0}^{m-1} \frac{f^{(0, k)}(x, c)}{k !}(y-c)^{k}-\sum_{i=0}^{n-1} \sum_{k=0}^{m-1} \frac{f^{(i, k)}(a, c)}{i ! k !}(x-a)^{i}(y-c)^{k} .
\end{gathered}
$$

Так как $f^{(n-1, m-1)}(x, y) \in A C(\bar{\Omega})$, то $f^{(n-1, m-1)}(a, y) \in A C([c, d])$, следовательно, $f^{(n-1,0)}(a, y) \in A C^{m}([c, d])$, откуда $f^{(i, 0)}(a, y) \in A C^{m}([c, d])(i=\overline{0, n-1})$. Используем лемму $[9$, c. 46$]$ :

$$
f^{(i, 0)}(a, y)=\frac{1}{(m-1) !} \int_{c}^{y}(y-c)^{m-1} \xi_{i}(y) d y+\sum_{k=0}^{m-1} \frac{f^{(i, k)}(a, c)}{k !}(y-c)^{k},
$$

где $\xi_{i}(y) \in L_{1}(c, d)$. Тогда

$$
\sum_{i=0}^{n-1} \frac{f^{(i, 0)}(a, y)}{i !}(x-a)^{i}=\sum_{i=0}^{n-1} \frac{(x-a)^{i}}{i !(m-1) !} \int_{c}^{y}(y-c)^{m-1} \xi_{i}(y) d y+\sum_{i=0}^{n-1} \sum_{k=0}^{m-1} \frac{f^{(i, k)}(a, c)}{i ! k !}(x-a)^{i}(y-c)^{k} .
$$

Аналогичным образом доказывается, что

$$
\sum_{k=0}^{m-1} \frac{f^{(0, k)}(x, c)}{k !}(y-c)^{k}=\sum_{k=0}^{m-1} \frac{(y-c)^{k}}{k !(n-1) !} \int_{a}^{x}(x-t)^{n-1} \psi_{k}(x) d x+\sum_{i=0}^{n-1} \sum_{k=0}^{m-1} \frac{f^{(i, k)}(a, c)}{i ! k !}(x-a)^{i}(y-c)^{k},
$$

где $\psi_{k}(y) \in L_{1}(a, b)$. Подставляя (20), (21) в (18), получим формулу (17), в которой

$$
d_{i k}=\frac{1}{i ! k !} f^{(i, k)}(a, c)
$$

Необходимость доказана.

Достаточность. Вычисляя непосредственно $\frac{\partial^{i+k} f}{\partial x^{i} \partial y^{k}}(0 \leq i<n, 0 \leq k<m)$, легко убедиться, что все они непрерывны в $\bar{\Omega}$, и

$$
\frac{\partial^{n+m-2} f}{\partial x^{n-1} \partial y^{m-1}}=\int_{a}^{x} \int_{c}^{y} \varphi(x, y) d x d y+\int_{a}^{x} \psi(x) d x+\int_{c}^{y} \xi(y) d y+(n-1) !(m-1) ! d_{n-1, m-1} .
$$

Очевидно, что $\frac{\partial^{n+m-2} f}{\partial x^{n-1} \partial y^{m-1}} \in A C(\bar{\Omega})$, откуда следует $f(x, y) \in A C^{n, m}(\bar{\Omega})$.

Теорема доказана полностью.

Заметим, что

$$
\begin{gathered}
\varphi(x, y)=f^{(n, m)}(x, y) \\
\psi_{k}(x)=f^{(n, k)}(x, c), k=\overline{0, m-1} \\
\xi_{i}(y)=f^{(i, m)}(a, y), i=\overline{0, n-1} \\
d_{i k}=\frac{1}{i ! k !} f^{(i, k)}(a, c) .
\end{gathered}
$$




\section{Смешанный дробный интеграл и смешанная дробная производная}

Определение 5 [1, с. 341]. Пусть $f(x, y) \in L_{1}(\Omega)$. Интеграл

$$
\left(I_{a+, c+}^{\alpha, \beta} f\right)(x, y)=\frac{1}{\Gamma(\alpha) \Gamma(\beta)} \int_{(a, x) \times(c, y)} \frac{f(t, s) d t d s}{(x-t)^{1-\alpha}(y-s)^{1-\beta}},
$$

где $\alpha>0, \beta>0$, называется смешанным дробным интегралом Римана-Лиувилля порядка $(\alpha, \beta)$.

Дробный интеграл (28), очевидно, определен на функциях $f(x, y) \in L_{1}(\Omega)$, существуя почти всюду.

С помощью теоремы Фубини доказывается полугрупповое свойство.

Пусть $f(x, y) \in L_{1}(a, b), \alpha, \beta, \gamma, \delta$ - положительные числа, тогда почти всюду в $\Omega$ справедливо равенство

$$
I_{a+, c+}^{\alpha, \beta} I_{a+, c+}^{\gamma, \delta} f=I_{a+, c+}^{\alpha+\gamma, \beta+\delta} f .
$$

Можно показать, что если $\alpha>0$, функция $f(x, y)$ определена в $\Omega$ и $f(x, y) \in L_{1}(\Omega)$, то

$$
\left(I_{a+, x}^{\alpha} f\right)(x, y) \in L_{1}(c, d) \quad \forall x \in(a, b) ; \quad\left(I_{a+, y}^{\alpha} f\right)(x, y) \in L_{1}(a, b) \quad \forall y \in(c, d) .
$$

В последних равенствах $I_{a+, x}^{\alpha} f, I_{a+, y}^{\alpha} f$ - частные дробные интегралы Римана-Лиувилля по переменным $x$ и $y$ соответственно.

С учетом этих равенств непосредственно проверяется, что

$$
\left(I_{a+, x}^{\alpha} I_{b+, y}^{\beta} f\right)(x, y)=\left(I_{b+, y}^{\beta} I_{a+, x}^{\alpha} f\right)(x, y)=\left(I_{a+, c+}^{\alpha, \beta} f\right)(x, y) .
$$

Определение 6 [1, с. 342]. Для функции $f(x, y)$, заданной на $\Omega$, выражение

$$
\left(D_{a+, c+}^{\alpha, \beta} f\right)(x, y)=\frac{1}{\Gamma(n-\alpha) \Gamma(m-\beta)} \frac{\partial^{n+m}}{\partial x^{n} \partial y^{m}} \int_{(a, x) \times(c, y)} \frac{f(t, s) d t d s}{(x-t)^{\alpha-n+1}(y-s)^{\beta-m+1}},
$$

где $\alpha>0, \beta>0$, называется смешанной дробной производной Римана-Лиувилля порядка $(\alpha, \beta), n=[\alpha]+1, m=[\beta]+1$.

Если функция $f(x, y)$ обладает свойством $I_{a+c+}^{n-\alpha, m-\beta} f \in A C^{n, m}(\bar{\Omega})$, то порядок взятия производных в (31) не имеет значения, и

$$
\left(D_{a+, c+}^{\alpha, \beta} f\right)(x, y) \in L_{1}(\Omega) .
$$

Определение 7 является двумерным аналогом определения 2.4 [1, с. 50].

Композиция смешанного дробного интеграла и смешанной дробной производной одного порядка. Следуя $[1$, c. 50], определим следующие классы функций.

Определение 7. Через $I_{a+, c+}^{\alpha, \beta}\left(L_{1}\right)$ обозначим класс функций $f(x, y)$, представимых смешанным дробным интегралом порядка $(\alpha, \beta)$ от суммируемой функции: $f=I_{a+, c+}^{\alpha, \beta} \varphi, \varphi \in L_{1}(\Omega)$.

Определение 8. Пусть $0<\alpha<1,0<\beta<1$. Будем говорить, что функция $f(x, y) \in L_{1}(a, b) \quad$ имеет суммируемую дробную производную $D_{a+, c+}^{\alpha, \beta} f, \quad$ ecли $I_{a+, c+}^{n-\alpha, m-\beta} f \in A C^{n, m}(\bar{\Omega})$.

Следующая теорема задает необходимое и достаточное условие однозначной разрешимости двухмерного интегрального уравнения Абеля.

Теорема 1. Для того чтобы $f(x, y) \in I_{a+, c+}^{\alpha, \beta}\left(L_{1}\right), \alpha>0, \beta>0$, необходимо и достаточно, чтобы

$$
f_{n-\alpha, m-\beta} \in A C^{n, m}(\bar{\Omega})
$$

где $n=[\alpha]+1, m=[\beta]+1$, и чтобы

$$
\begin{gathered}
f_{n-\alpha, m-\beta}^{(i, 0)}(a, y) \equiv 0, \quad i=\overline{0, n-1} ; \\
f_{n-\alpha, m-\beta}^{(0, k)}(x, c) \equiv 0, \quad k=\overline{0, m-1} ; \\
f_{n-\alpha, m-\beta}^{(i, k)}(a, c)=0, \quad i=\overline{0, n-1}, \quad k=\overline{0, m-1} .
\end{gathered}
$$

Доказательство. Необходимость. Пусть $f=I_{a+, c+}^{\alpha, \beta} \varphi, \varphi \in L_{1}(\Omega)$. Тогда в силу полугруппового свойства 


$$
f_{n-\alpha, m-\beta}(x, y)=I_{a+, c+}^{n-\alpha, m-\beta} f=I_{a+, c+}^{n, m} \varphi,
$$

где $\varphi \in L_{1}(\Omega)$. Отсюда следует выполнимость условий (33)-(35). Выполнимость условия (32) вытекает из леммы 4.

Достаточность. При выполнении условия (32) можем представить $f_{n-\alpha, m-\beta}$ согласно лемме 3 в виде

$$
\begin{gathered}
f_{n-\alpha, m-\beta}(x, y)=\frac{1}{(n-1) !(m-1) !} \int_{a}^{x} \int_{c}^{y}(x-t)^{n-1}(y-s)^{m-1} f_{n-\alpha, m-\beta}^{(n, m)}(x, y) d x d y+ \\
+\sum_{i=0}^{n-1} \frac{f^{(i, 0)}(a, y)}{i !}(x-a)^{i}+\sum_{k=0}^{m-1} \frac{f_{n-\alpha, m-\beta}^{(0, k)}(x, c)}{k !}(y-c)^{k}-\sum_{i=0}^{n-1} \sum_{k=0}^{m-1} \frac{f_{n-\alpha, m-\beta}^{(i, k)}(a, c)}{i ! k !}(x-a)^{i}(y-c)^{k},
\end{gathered}
$$

где $f_{n-\alpha, m-\beta}^{(n, m)} \in L_{1}(\Omega)$. С учетом условий (33) - (35) последнее равенство запишется в виде

$$
f_{n-\alpha, m-\beta}(x, y)=\frac{1}{(n-1) !(m-1) !} \int_{a c}^{x} \int_{c}^{y}(x-t)^{n-1}(y-s)^{m-1} f_{n-\alpha, m-\beta}^{(n, m)}(x, y) d x d y .
$$

Используя полугрупповое свойство (29), можно записать

$$
I_{a+, c+}^{n-\alpha, m-\beta} f=I_{a+, c+}^{n, m} f_{n-\alpha, m-\beta}^{(n, m)}=I_{a+, c+}^{n-\alpha, m-\beta} I_{a+, c+}^{\alpha, \beta} f_{n-\alpha, m-\beta}^{(n, m)} .
$$

Отсюда $I_{a+, c+}^{n-\alpha, m-\beta}\left(f-I_{a+, c+}^{\alpha, \beta} f_{n-\alpha, m-\beta}^{(n, m)}\right)=0$. Применив к этому равенству интеграл $I_{a+, c+}^{\alpha, \beta}$, получим

$$
I_{a+, c+}^{n, m}\left(f-I_{a+, c+}^{\alpha, \beta} f_{n-\alpha, m-\beta}^{(n, m)}\right) d x d y=0 .
$$

Отсюда $f=I_{a+, c+}^{\alpha, \beta} f_{n-\alpha, m-\beta}^{(n, m)}, f_{n-\alpha, m-\beta}^{(n, m)} \in L_{1}(\Omega)$. Теорема доказана.

Отметим, что теорема 1 является обобщением теоремы 2.3 [1, с. 49] на случай двух переменных. Из нее, в частности, следует, что класс функций, имеющих суммируемую дробную производную $D_{a+, c+}^{\alpha, \beta} f$ в смысле определения 8, шире класса функций $I_{a+, c_{+}}^{\alpha, \beta}\left(L_{1}\right)$. А именно, классу $I_{a+, c+}^{\alpha, \beta}\left(L_{1}\right)$ принадлежат только те функции, имеющие суммируемую дробную производную $D_{a+, c+}^{\alpha, \beta} f$, для которых выполняются равенства (33)-(35).

Теорема 2. Пусть $\alpha>0, \beta>0$. Тогда равенство

$$
D_{a+, c+}^{\alpha, \beta} I_{a+, c+}^{\alpha, \beta} f=f(x, y)
$$

выполняется для любой суммируемой функции $f(x, y)$.

Доказательство. Имеем

$$
\begin{gathered}
D_{a+, c+}^{\alpha, \beta} I_{a+, c+}^{\alpha, \beta} f=\frac{\partial^{n+m}}{\partial x^{n} \partial y^{m}} I_{a+, c+}^{n-\alpha, m-\beta} I_{a+, c+}^{\alpha, \beta} f=\frac{1}{\Gamma(\alpha) \Gamma(\beta) \Gamma(n-\alpha) \Gamma(m-\beta)} \times \\
\times \frac{\partial^{n+m}}{\partial x^{n} \partial y^{m}} \int_{a}^{x} \int_{c}^{y} \frac{d t d s}{(x-t)^{\alpha}(y-s)^{\beta}} \int_{a}^{t} \int_{c}^{s} \frac{f(u, v) d u d v}{(t-u)^{n-\alpha}(s-v)^{m-\beta}} .
\end{gathered}
$$

Меняя порядок интегрирования, получим

$$
\begin{aligned}
& D_{a+, c+}^{\alpha, \beta} I_{a+, c+}^{\alpha, \beta} f=\frac{1}{\Gamma(\alpha) \Gamma(\beta) \Gamma(n-\alpha) \Gamma(m-\beta)} \times \\
& \times \frac{\partial^{n+m}}{\partial x^{n} \partial y^{m}} \int_{a}^{x} \int_{c}^{y} f(u, v) d u d v \int_{u}^{x} \int_{v}^{y} \frac{d t d s}{(t-u)^{n-\alpha}(s-v)^{m-\beta}(x-t)^{\alpha}(y-s)^{\beta}}= \\
& =\frac{\partial^{n+m}}{\partial x^{n} \partial y^{m}} \int_{a}^{x} \int_{c}^{y} f(u, v) d u d v \frac{1}{\Gamma(\alpha) \Gamma(n-\alpha)} \int_{u}^{x} \frac{d t}{(t-u)^{n-\alpha}(x-t)^{\alpha}} \times \\
& \times \frac{1}{\Gamma(\beta) \Gamma(m-\beta)} \int_{v}^{y} \frac{d s}{(s-v)^{m-\beta}(y-s)^{\beta}}= \\
& =\frac{1}{\Gamma(n) \Gamma(m)} \frac{\partial^{n+m}}{\partial x^{n} \partial y^{m}} \int_{a}^{x} \int_{c}^{y} f(u, v)(x-u)^{n-1}(y-v)^{m-1} d u d v=f(x, y),
\end{aligned}
$$

что и требовалось доказать.

Теорема 3. Для любой функции $f(x, y) \in I_{a+, c+}^{\alpha, \beta}\left(L_{1}\right)$ выполняется равенство

$$
I_{a+, c+}^{\alpha, \beta} D_{a+, c+}^{\alpha, \beta} f=f(x, y),
$$


а для любой функции, имеющей суммируемую производную $D_{a+, c+}^{\alpha, \beta} f$ (в смысле определения 8), выполняется равенство

$$
\begin{gathered}
I_{a+, c+}^{\alpha, \beta} D_{a+, c+}^{\alpha, \beta} f=f(x, y)-\sum_{i=0}^{n-1} \frac{(x-a)^{\alpha-i-1}}{\Gamma(\alpha-i)} f_{n-\alpha, 0}^{(n-i-1,0)}(a, y)- \\
-\sum_{k=0}^{m-1} \frac{(y-c)^{\beta-k-1}}{\Gamma(\beta-k)} f_{0, m-\beta}^{(0, m-k-1)}(x, c)+\sum_{i=0}^{n-1} \sum_{k=0}^{m-1} \frac{(x-a)^{\alpha-i-1}(y-c)^{\beta-k-1}}{\Gamma(\alpha-i) \Gamma(\beta-k)} f_{n-\alpha, m-\beta}^{(n-i-1, m-k-1)}(a, c),
\end{gathered}
$$

где $f_{\gamma, \delta}(x, y)=I_{a+, c+}^{\gamma, \delta} f$.

Доказательство. Пусть $f(x, y) \in I_{a+, c+}^{\alpha, \beta}\left(L_{1}\right)$, тогда $f(x, y)=I_{a+, c+}^{\alpha, \beta} \varphi, \varphi(x, y) \in L_{1}(\Omega)$. На основании теоремы 2 имеем

$$
I_{a+, c+}^{\alpha, \beta} D_{a+, c+}^{\alpha, \beta} f=I_{a+, c+}^{\alpha, \beta} D_{a+, c+}^{\alpha, \beta} I_{a+, c+}^{\alpha, \beta} \varphi=I_{a+, c+}^{\alpha, \beta} \varphi=f(x, y) .
$$

Пусть теперь $I_{a+, c+}^{1-\alpha, \beta} f \in A C(\bar{\Omega})$. Согласно лемме 3 , интеграл $f_{n-\alpha, m-\beta}(x, y)=I_{a+, c+}^{n-\alpha, \beta} f$ представим в виде

$$
\begin{gathered}
f_{n-\alpha, m-\beta}(x, y)=I_{a+, c+}^{n, m} f_{n-\alpha, m-\beta}^{(n, m)}+\sum_{i=0}^{n-1} \frac{f_{n-\alpha, m-\beta}^{(i, 0)}(a, y)}{i !}(x-a)^{i}+ \\
+\sum_{k=0}^{m-1} \frac{f_{n-\alpha, m-\beta}^{(0, k)}(x, c)}{k !}(y-c)^{k}-\sum_{i=0}^{n-1} \sum_{k=0}^{m-1} \frac{f_{n-\alpha, m-\beta}^{(i, k)}(a, c)}{i ! k !}(x-a)^{i}(y-c)^{k} .
\end{gathered}
$$

В силу полугруппового свойства выполняется равенство

$$
I_{a+, c+}^{n, m} f_{n-\alpha, m-\beta}^{(n, m)}=I_{a+, c+}^{n-\alpha, m-\beta} I_{a+, c+}^{\alpha, \beta} f_{n-\alpha, m-\beta}^{(n, m)} .
$$

Далее,

$$
\begin{gathered}
\frac{(x-a)^{i}}{i !} f_{n-\alpha, m-\beta}^{(i, 0)}(a, y)=I_{a+, c+}^{n-\alpha, m-\beta}\left(D_{a+, x}^{n-\alpha} \frac{(x-a)^{i}}{i !} D_{c+, y}^{m-\beta} f_{n-\alpha, m-\beta}^{(i, 0)}(a, y)\right)+\frac{(x-a)^{i}(y-c)^{m-\beta-1}}{i ! \Gamma(m-\beta)} f_{n-\alpha, 1}^{(i, 0)}(a, c)= \\
=I_{a+, c+}^{n-\alpha, m-\beta}\left(\frac{(x-a)^{i-n+\alpha}}{\Gamma(1+i-n+\alpha)} f_{n-\alpha, 0}^{(i, 0)}(a, y)\right)+\frac{(x-a)^{i}(y-c)^{m-\beta-1}}{i ! \Gamma(m-\beta)} f_{n-\alpha, 1}^{(i, 0)}(a, c) .
\end{gathered}
$$

Из последнего равенства следует, что

$$
\begin{gathered}
\sum_{i=0}^{n-1} \frac{f_{n-\alpha, m-\beta}^{(i, 0)}(a, y)}{i !}(x-a)^{i}= \\
=I_{a+, c+}^{n-\alpha, m-\beta}\left(\sum_{i=0}^{n-1} \frac{(x-a)^{i-n+\alpha}}{\Gamma(1+i-n+\alpha)} f_{n-\alpha}^{(i, 0)}(a, y)\right)+\sum_{i=0}^{n-1} \frac{(x-a)^{i}(y-c)^{m-\beta-1}}{i ! \Gamma(m-\beta)} f_{n-\alpha, 1}^{(i, 0)}(a, c),
\end{gathered}
$$

откуда, переобозначая индекс суммирования, получаем

$$
\begin{gathered}
\sum_{i=0}^{n-1} \frac{f_{n-\alpha, m-\beta}^{(i, 0)}(a, y)}{i !}(x-a)^{i}= \\
=I_{a+, c+}^{n-\alpha, m-\beta}\left(\sum_{i=0}^{n-1} \frac{(x-a)^{\alpha-i-1}}{\Gamma(\alpha-i)} f_{n-\alpha, 0}^{(n-i-1,0)}(a, y)\right)+\sum_{i=0}^{n-1} \frac{(x-a)^{i}(y-c)^{m-\beta-1}}{i ! \Gamma(m-\beta)} f_{n-\alpha, 1}^{(i, 0)}(a, c) .
\end{gathered}
$$

Аналогично получается равенство

$$
\begin{gathered}
\sum_{k=0}^{m-1} \frac{f_{n-\alpha, m-\beta}^{(0, k)}(x, c)}{k !}(y-c)^{k}= \\
=I_{a+, c+}^{n-\alpha, m-\beta}\left(\sum_{k=0}^{m-1} \frac{(y-c)^{\beta-k-1}}{\Gamma(\beta-k)} f_{0, m-\beta}^{(0, m-k-1)}(x, c)\right)+\sum_{k=0}^{m-1} \frac{(y-c)^{k}(x-a)^{n-\alpha-1}}{k ! \Gamma(n-\alpha)} f_{1, m-\beta}^{(0, k)}(a, c) .
\end{gathered}
$$

Нетрудно видеть, что

$$
\begin{gathered}
\sum_{i=0}^{n-1} \sum_{k=0}^{m-1} \frac{f_{n-\alpha, m-\beta}^{(i, k)}(a, c)}{i ! k !}(x-a)^{i}(y-c)^{k}=I_{a+, c+}^{n-\alpha, \beta}\left(\sum_{i=0}^{n-1} \sum_{k=0}^{m-1} I_{a+, c+}^{n-\alpha, m-\beta}\left(\frac{(x-a)^{i}(y-c)^{k}}{i ! k !}\right) f_{n-\alpha, m-\beta}^{(i, k)}(a, c)\right)= \\
=I_{a+, c+}^{n-\alpha, m-\beta}\left(\sum_{i=0}^{n-1} \sum_{k=0}^{m-1} \frac{(x-a)^{\alpha-i-1}(y-c)^{\beta-k-1}}{\Gamma(\alpha-i) \Gamma(\beta-k)} f_{n-\alpha, m-\beta}^{(n-i-1, m-k-1)}(a, c)\right) .
\end{gathered}
$$

С учетом равенств (48), (51)-(53) равенство (47) запишется в виде 


$$
\begin{gathered}
I_{a+, c+}^{n-\alpha, m-\beta} f=I_{a+, c+}^{n-\alpha, m-\beta} I_{a+, c+}^{\alpha, \beta} D_{a+, c+}^{\alpha, \beta} f+I_{a+, c+}^{n-\alpha, m-\beta}\left(\sum_{i=0}^{n-1} \frac{(x-a)^{\alpha-i-1}}{\Gamma(\alpha-i)} f_{n-\alpha, 0}^{(n-i-1,0)}(a, y)\right)+\sum_{i=0}^{n-1} \frac{(x-a)^{i}(y-c)^{m-\beta-1}}{i ! \Gamma(m-\beta)} f_{n-\alpha, 1}^{(i, 0)}(a, c)+ \\
+I_{a+, c+}^{n-\alpha, \beta-\beta}\left(\sum_{k=0}^{m-1} \frac{(y-c)^{\beta-k-1}}{\Gamma(\beta-k)} f_{0, m-\beta}^{(0, m-k-1)}(x, c)\right)+\sum_{k=0}^{m-1} \frac{(y-c)^{k}(x-a)^{n-\alpha-1}}{k ! \Gamma(n-\alpha)} f_{1, m-\beta}^{(0, k)}(a, c)- \\
\quad-I_{a+, c+}^{n-\alpha, m-\beta}\left(\sum_{i=0}^{n-1} \sum_{k=0}^{m-1} \frac{(x-a)^{\alpha-i-1}(y-c)^{\beta-k-1}}{\Gamma(\alpha-i) \Gamma(\beta-k)} f_{n-\alpha, m-\beta}^{(n-i-1, m-k-1)}(a, c)\right)
\end{gathered}
$$

Группируя слагаемые, получим

$$
\begin{aligned}
& I_{a+, c+}^{n-\alpha, m-\beta}\left(f-I_{a+, c+}^{\alpha, \beta} D_{a+, c+}^{\alpha, \beta} f-\sum_{i=0}^{n-1} \frac{(x-a)^{\alpha-i-1}}{\Gamma(\alpha-i)} f_{n-\alpha, 0}^{(n-i-1,0)}(a, y)-\sum_{k=0}^{m-1} \frac{(y-c)^{\beta-k-1}}{\Gamma(\beta-k)} f_{0, m-\beta}^{(0, m-k-1)}(x, c)+\right. \\
& \left.+\sum_{i=0}^{n-1} \sum_{k=0}^{m-1} \frac{(x-a)^{\alpha-i-1}(y-c)^{\beta-k-1}}{\Gamma(\alpha-i) \Gamma(\beta-k)} f_{n-\alpha, m-\beta}^{(n-i-1, m-k-1)}(a, c)\right)= \\
& \quad=\sum_{i=0}^{n-1} \frac{(x-a)^{i}(y-c)^{m-\beta-1}}{i ! \Gamma(m-\beta)} f_{n-\alpha, 1}^{(i, 0)}(a, c)+\sum_{k=0}^{m-1} \frac{(y-c)^{k}(x-a)^{n-\alpha-1}}{k ! \Gamma(n-\alpha)} f_{1, m-\beta}^{(0, k)}(a, c) .
\end{aligned}
$$

В правой части равенства (55) под интегралом - суммируемая функция. Применяя к обоим частям равенства (55) оператор $I_{a+, c_{+}}^{\alpha, \beta}$, получим

$$
\begin{gathered}
I_{a+, c+}^{n, m}\left(f-I_{a+, c+}^{\alpha, \beta} D_{a+, c+}^{\alpha, \beta} f-\sum_{i=0}^{n-1} \frac{(x-a)^{\alpha-i-1}}{\Gamma(\alpha-i)} f_{n-\alpha, 0}^{(n-i-1,0)}(a, y)-\sum_{k=0}^{m-1} \frac{(y-c)^{\beta-k-1}}{\Gamma(\beta-k)} f_{0, m-\beta}^{(0, m-k-1)}(x, c)+\right. \\
\left.+\sum_{i=0}^{n-1} \sum_{k=0}^{m-1} \frac{(x-a)^{\alpha-i-1}(y-c)^{\beta-k-1}}{\Gamma(\alpha-i) \Gamma(\beta-k)} f_{n-\alpha, m-\beta}^{(n-i-1, m-k-1)}(a, c)\right)= \\
=\sum_{i=0}^{n-1} \frac{(x-a)^{i+\alpha}(y-c)^{m-1}}{\Gamma(i+\alpha+1) \Gamma(m)} f_{n-\alpha, 1}^{(i, 0)}(a, c)+\sum_{k=0}^{m-1} \frac{(y-c)^{k+\beta}(x-a)^{n-1}}{\Gamma(k+\beta+1) \Gamma(n)} f_{1, m-\beta}^{(0, k)}(a, c) .
\end{gathered}
$$

Под интегралом в левой части равенства стоит суммируемая функция, а правая часть равенства абсолютно непрерывна. Находя смешанную производную $\frac{\partial^{m+n}}{\partial x^{n} \partial y^{m}}$ от обоих частей равенства, получим

$$
\begin{gathered}
f-I_{a+, c+}^{\alpha, \beta} D_{a+, c+}^{\alpha, \beta} f-\sum_{i=0}^{n-1} \frac{(x-a)^{\alpha-i-1}}{\Gamma(\alpha-i)} f_{n-\alpha, 0}^{(n-i-1,0)}(a, y)-\sum_{k=0}^{m-1} \frac{(y-c)^{\beta-k-1}}{\Gamma(\beta-k)} f_{0, m-\beta}^{(0, m-k-1)}(x, c)+ \\
+\sum_{i=0}^{n-1} \sum_{k=0}^{m-1} \frac{(x-a)^{\alpha-i-1}(y-c)^{\beta-k-1}}{\Gamma(\alpha-i) \Gamma(\beta-k)} f_{n-\alpha, m-\beta}^{(n-i-1, m-k-1)}(a, c)=0 .
\end{gathered}
$$

Теорема доказана.

Заключение. Доказанные в работе лемма 3 о представимости функции $f(x, y) \in A C^{m, n}(\bar{\Omega})$ в виде (6) и лемма 4 обобщают известные ранее леммы 1 и 2 на двумерный случай. Леммы 3,4 позволяют доказать теорему 1 (необходимое и достаточное условие представимости функции $f(x, y)$ в виде смешанного дробного интеграла от суммируемой функции) и теоремы 2,3 о композиции смешанного дробного интеграла и смешанной дробной производной. Отметим, что теоремы 2,3 обобщают результаты теоремы $2.4[1$, с. 50$]$ на двухмерный случай.

\section{БИБЛИОГРАФИЧЕСКИЙ СПИСОК}

1. Самко С. Г., Килбас А. А., Маричев О. И. Интегралы и производные дробного порядка и их приложения. Минск: Наука и техника, 1987. 702 с.

2. 2. Oldham K.B., Spanier J. The Fractional Calculus. New York-London: Academic Press, 1974. 234 p.

3. Podlubny I. Fractional Differential Equations. //Mathematics is Sciences and Engineering, Vol. 198, San-Diego: Academic Press, 1999. P. 32-39.

4. Нахушев А.М. Уравнения математической биологии. М: Высш. шк., 1995. $301 \mathrm{c.}$

5. Нахушев А.М. Элесенты дробного исчисления и их применение. Нальчик: КБНЦ РАН, 2000. 299 с.

6. Нахушева B.A. Некоторые классы дифференциальных уравнений математических моделей нелокальных физических процессов. Нальчик: КБНЦ РАН, 2002. 100 с.

7. Metzler R., Klafter J. The randomwalk's guide to anomalous diffusion: a fractional dynamics approac //Phys. Reports. 2000. Vol. 339. P. 1-77.

8. Sokolov I.M., Klafter J., Blumen A.Fractional kinetics // Physics Today, November 2002. P. 48-54.

9. Kilbas A.A., Srivastava H.M., Trujillo J.J. Fractional differential equations: An emergent field in applied and mathe- 
matical sciences// Factorization, Singular Operators and Related Problems. Proceedings of the Conference in Honour of Professor Georgii Litvinchuk. Eds.: Samko, Stefan; Lebre, Anarino and Santos, Antonio. Kluwer, Dordrecht-BostonLondon, 2003. P. 151-173.

10. Love E.R. Some integral equations involving hypergeometric functions// Proc. Edinburgh Math. Soc., 1967. Vol. 15. №3. P. 169-198.

11. Saigo M. A remark on integral operators involving the Gauss hypergeometric function// Math. Rep. Kyushu Univ., 1978. Vol. 11. №2. P. 135-143.

12. Килбас A.A., Репин О.A. Нелокальная задача для уравнения смешанного типа с частной производной РиманаЛиувилля и операторами обобщенного дробного интегрирования в краевом условии// Минск: Труды Института Математики БАН, 2004. Т.12. №2. С. 75-81.

13. Репин О.А. Краевые задачи со смещением для уравнений гиперболического и смешанного типов. Самара: Издво Саратовск. ун-та (Самарск. филиал), 1992. 162 с.

14. Андреев A. А. Об одном обобщении операторов дробного интегродифференцирования и его приложениях // Интегральные уравнения и краевые задачи математической физики. Матер. Всесоюзной конф. Владивосток. 1990. C. 91.

15. Андреев А.А., Огородников Е.Н. Матричные интегродифференциальные операторы и их применение // Вестник Самарск. гос. техн. ун-та. Сер.: физ.-мат. науки. Вып. 7. Самара: СамГТУ, 1999. С. 27-37.

16. Еремин A. C., Андреев A. A. Краевая задача для уравнения с матричным интегродифференциальным оператором // Вестник Самарск. гос. техн. ун-та. Сер.: физ.-мат. науки. Вып. 26. Самара: СамГТУ, 2004. С. 5-11.

17. Vasilache $S$. Asupra unei ecuatii integrale de tip Abel cu doua variabile // Comun. Acad. R.P. Romane, 1953. Vol. 3. №3-4. P. 109-113.

18. Смирнов В. И. Курс высшей математики. Т. 5. М.: ОГИЗ, 1947. 584 с.

19. Колмогоров А.Н., Фомин С.В. Элементы теории функций и функционального анализа. М: Наука, 1968. 496 с.

20. Тер-Крикоров А. М., Шабунин М. И. Курс математического анализа: Учеб. пособие для вузов. М.: Наука, 1988. $816 \mathrm{c.}$

Поступила 15.12.2004 2.

УдК 517.956

\section{Е.Н. Огородников, Е.Ю. Арланова}

\section{НЕКОТОРЫЕ НЕЛОКАЛЬНЫЕ АНАЛОГИ ЗАДАЧИ КОШИ-ГУРСА И СУЩЕСТВЕННО НЕЛОКАЛЬНЫЕ КРАЕВЫЕ ЗАДАЧИ ДЛЯ СИСТЕМЫ УРАВНЕНИЙ БИЦАДЗЕ-ЛЫКОВА В СПЕЦИАЛЬНЫХ СЛУЧЯХ}

На примере уравнения влагопереноса и системы подобных уравнений в условиях отсутствия единственности решения задачи Коши-Гурса рассмотрены простейшие нелокальные аналоги этой задачи и некоторые существенно нелокальные краевые задачи с условиями типа Бицадзе-Самарского и Бицадзе-Нахушева. Обоснована их корректность.

Введение. Уравнение

$$
y^{2} u_{x x}-u_{y y}+a u_{x}=0
$$

описывающее при $a>0$ процесс переноса потока влаги в капиллярно-пористых средах выведено в 1965 г. А. В. Лыковым методами термодинамики необратимых процессов и с тех пор известно как уравнение влагопереноса [1]. Ранее в 1959 г. в монографии А. В. Бицадзе [2] это уравнение приводилось в качестве примера, для которого при $|a| \leq 1$ корректна по Адамару задача Коши

$$
\lim _{y \rightarrow+0} u(x, 0)=\tau(x), \lim _{y \rightarrow+0} u_{y}(x, y)=v(x), x \in(0,1)
$$

с начальными данными на линии $y=0$ параболического вырождения, хотя и нарушено известное условие Геллерстедта [3]. Регулярное в области $\Omega=\left\{(x, y): 0<x-\frac{y^{2}}{2}<x+\frac{y^{2}}{2}<1\right\}$ решение задачи (2) для уравнения (1) находится методом Римана и при $|a|<1$ имеет вид [2,4]:

$$
u(x, y)=B(\alpha, \beta) y \int_{x-\frac{y^{2}}{2}}^{x+\frac{y^{2}}{2}} \tau(s)\left(s-x+\frac{y^{2}}{2}\right)^{\beta-1}\left(x+\frac{y^{2}}{2}-s\right)^{\alpha-1} d s+
$$

